\title{
MicroRNA-584 directly targets CCND1 and inhibits cell proliferation and invasion in pancreatic cancer
}

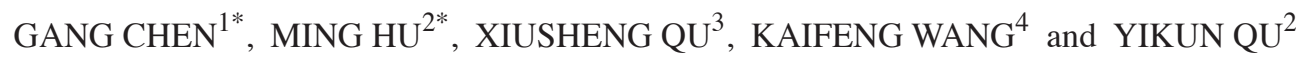 \\ Departments of ${ }^{1}$ Gastroenterology, ${ }^{2}$ General Surgery, ${ }^{3}$ Radiochemotherapy and ${ }^{4}$ Vascular Surgery, \\ First Affiliated Hospital of Jiamusi University, Jiamusi, Heilongjiang 154003, P.R. China
}

Received March 19, 2018; Accepted October 16, 2018

DOI: $10.3892 / \mathrm{mmr} .2018 .9651$

\begin{abstract}
Multiple previous studies have demonstrated that the dysregulation of microRNAs (miRNAs) is implicated in the occurrence and development of pancreatic cancer. Therefore, a further characterisation of deregulated miRNAs in pancreatic cancer may provide novel insight into the oncogenesis and progression of pancreatic cancer, which may facilitate the identification of effective therapeutic targets for treating patients with this disease. In the present study, reverse transcription-quantitative polymerase chain reaction analysis demonstrated that the expression level of miRNA-584-5p (miR-584) was significantly decreased in pancreatic cancer tissues and cell lines. It was demonstrated that restoration of miR-584 expression significantly suppressed the proliferative and invasive ability of pancreatic cancer cells. Bioinformatics analysis predicted that cyclin D1 (CCND1) was a putative target of miR-584. Subsequent experiments demonstrated that CCND1 was a direct target gene of miR-584 in pancreatic cancer cells. Furthermore, the inhibition of CCND1 mimicked the suppressive effect of miR-584 overexpression in pancreatic cancer cells. The restoration of CCND1 expression significantly abolished the inhibitory effects of miR-584 overexpression on pancreatic cancer cells. Collectively, the present results demonstrated that miR-584 inhibited the development of pancreatic cancer by directly targeting CCND1, suggesting that this miRNA may represent a potential therapeutic target for this fatal disease.
\end{abstract}

\section{Introduction}

Pancreatic cancer, a highly lethal malignancy, is the sixth leading cause of cancer-associated mortalities due to malignant

Correspondence to: Professor Yikun Qu, Department of General Surgery, First Affiliated Hospital of Jiamusi University, 348 Dexiang Road, Jiamusi, Heilongjiang 154003, P.R. China

E-mail: qu_yikun@126.com

*Contributed equally

Key words: pancreatic cancer, microRNA-584, proliferation, invasion, cyclin D1 tumours in China (1). Pancreatic cancer is difficult to diagnose early since the clinical symptoms are not obvious; therefore, patients with pancreatic cancer are frequently diagnosed at advanced stages and are unsuitable for surgical resection (2). Despite significant developments in diagnosis and therapy, the treatment outcomes of patients with pancreatic cancer remain poor with high recurrence and an unsatisfactory five-year survival rate (3). Numerous genetic and epigenetic alterations that cause inactivation of tumour suppressor genes and activation of oncogenes, contribute to pancreatic cancer pathogenesis and development $(4,5)$. However, the causes of pancreatic cancer remain largely unknown. Therefore, further elucidation of the underlying mechanisms of pancreatic cancer initiation and progression is urgently required to identify effective therapeutic strategies for the treatment of patients with this disease.

MicroRNAs (miRNAs) are a class of highly conserved and non-coding RNA molecules that are involved in the regulation of gene expression (6). miRNAs negatively modulate gene expression by recognising and directly binding to the 3'-untranslated regions (3'-UTRs) of their target genes, inhibiting translation and/or inducing mRNAs degradation (7). miRNAs are aberrantly expressed in numerous human malignancy types, including pancreatic (8), gastric (9), thyroid (10) and lung (11) cancer. By modulating the expression of their targets, miRNAs affect numerous biological processes and thus are involved in the regulation of tumourigenesis and tumour progression $(12,13)$. Specifically, miRNAs that are downregulated in human cancer may serve tumour suppressive roles (14). By contrast, oncogenic miRNAs are generally upregulated and serve oncogenic roles during carcinogenesis and cancer progression (15). Therefore, understanding the expression patterns, biological roles and the mechanisms associated with miRNA regulation is crucial for anticancer therapy.

miR-584-5p (miR-584) serves tumour suppressive roles in multiple types of human malignancy (16-18). However, the expression pattern and detailed roles of miR-584 in pancreatic cancer and its underlying mechanisms require further examination and investigation. In the present study, it was demonstrated that miR-584 is downregulated in pancreatic cancer. In addition, miR-584 inhibited cell proliferation and invasion in pancreatic cancer by directly targeting cyclin D1 (CCND1). 


\section{Materials and methods}

Tissue collection. A total of 26 primary pancreatic cancer tissues and their matched normal adjacent specimens were collected from patients (17 males, 9 females; age, 42-67 years) The First Affiliated Hospital of Jiamusi University (Jiamusi, China) between June 2014 and February 2016. None of these patients had received chemotherapy, radiotherapy or other treatments prior to surgical resection. Patients that had been treated with chemotherapy, radiotherapy or other treatments prior to surgery were excluded from the study. All tissue specimens were quickly snap-frozen in liquid nitrogen subsequent to surgical resection and were stored at $-80^{\circ} \mathrm{C}$. The present study was approved by The Ethics Committee of the First Affiliated Hospital of Jiamusi University and was performed in accordance with the Declaration of Helsinki and the guidelines of the Ethics Committee of The First Affiliated Hospital of Jiamusi University. Informed written consent was obtained from all patients enrolled in the present study.

Cell culture. A normal human pancreatic cell line HPDE6c7 was purchased from The American Type Culture Collection (Manassas, VA, USA). A total of three human pancreatic cancer cell lines, Panc-1, Sw1990 and Bxpc-3, were purchased from Shanghai Institute of Biochemistry and Cell Biology (Shanghai, China). All these cell lines were cultured at $37^{\circ} \mathrm{C}$ under $5 \% \mathrm{CO}_{2}$, and were maintained in Dulbecco's modified Eagle's medium (DMEM) supplemented with $10 \% \mathrm{v} / \mathrm{v}$ fetal bovine serum (FBS), $100 \mathrm{U} / \mathrm{ml}$ penicillin and $100 \mathrm{U} / \mathrm{ml}$ streptomycin (all from Gibco; Thermo Fisher Scientific, Inc., Waltham, MA, USA).

Transfection. miR-584 mimics, negative control miRNA mimics (miR-NC), small interfering RNA (siRNA) targeting CCND1 (si-CCND1) and negative control siRNA (si-NC) were chemically synthesised by Guangzhou RiboBio Co., Ltd. (Guangzhou, China). The miR-584 mimics sequence was 5'-UUAUGGUUU GCCUGGGACUGAG-3' and the miR-NC sequence was 5'-UUC UCCGAACGUGUCACGUTT-3'. The si-CCND1 sequence was 5'-CUACCUGGACCGUUUCUUGUU-3' and the si-NC sequence was 5'-UUCUCCGAACGUGUCACGUUU-3'. The cytomegalovirus promoter (pCMV)-CCND1 and empty pCMV plasmids were ordered from Amspring (Changsha, China). Cells were seeded $\left(7 \times 10^{5}\right.$ cells/well) into six-well plates and cultured to $60-70 \%$ confluence. Prior to transfection, the culture medium was replaced with fresh FBS-free DMEM. Cells were transfected with miR-584 mimics (100 pmol), miR-NC (100 pmol), si-CCND1 (100 pmol), si-NC (100 pmol), pCMV-CCDN1 (4 $\mu \mathrm{g})$ or pCMV $(4 \mu \mathrm{g})$ using Lipofectamine ${ }^{\circledR} 2000$ Reagent (Invitrogen; Thermo Fisher Scientific, Inc.), according to the manufacturer's protocol. Transfected cells were incubated at $37^{\circ} \mathrm{C}$ with $5 \% \mathrm{CO}_{2}$ for $8 \mathrm{~h}$. Subsequently, the culture medium was replaced with $10 \% \mathrm{v} / \mathrm{v}$ FBS-containing DMEM. The efficiency of miR-584 mimics and pCMV-CCND1 transfection was determined using reverse transcription-quantitative polymerase chain reaction (RT-qPCR) and western blot analysis, respectively.

$R T-q P C R$. Total RNA of the tissues or cultured cells was isolated using TRIzol ${ }^{\circledR}$ (Thermo Fisher Scientific, Inc.) reagent, according to the manufacturer's protocol. For the analysis of miR-584 expression, synthesis of miR-584 cDNA was conducted using a TaqMan ${ }^{\mathrm{TM}}$ miRNA RT kit (Applied Biosystems; Thermo Fisher Scientific, Inc.) with the following parameters: $16^{\circ} \mathrm{C}$ for $30 \mathrm{~min}, 42^{\circ} \mathrm{C}$ for $30 \mathrm{~min}$ and $85^{\circ} \mathrm{C}$ for $5 \mathrm{~min}$. Subsequently, qPCR was conducted using the Applied Biosystems 7500 Sequence Detection System (Thermo Fisher Scientific, Inc.) using a TaqMan miRNA PCR kit (Applied Biosystems; Thermo Fisher Scientific, Inc.) and qPCR was performed as follows: $50^{\circ} \mathrm{C}$ for $2 \mathrm{~min}, 95^{\circ} \mathrm{C}$ for $10 \mathrm{~min}$; followed by 40 cycles of denaturation at $95^{\circ} \mathrm{C}$ for $15 \mathrm{sec}$; and annealing/extension at $60^{\circ} \mathrm{C}$ for $60 \mathrm{sec}$. For the detection of CCND1 mRNA expression, total RNA was subjected to RT for the synthesis of cDNA using a PrimeScript RT Reagent kit (Takara Biotechnology Co., Ltd., Dalian, China) as follows: $37^{\circ} \mathrm{C}$ for $15 \mathrm{~min}$ and $85^{\circ} \mathrm{C}$ for $5 \mathrm{sec}$. A SYBR Premix Ex Taq kit (Takara Biotechnology Co., Ltd.) was used to conduct the PCR amplification. qPCR was performed with thermocycling conditions as follows: initial denaturation for $5 \mathrm{~min}$ at $95^{\circ} \mathrm{C}$, followed by 40 cycles of $95^{\circ} \mathrm{C}$ for $30 \mathrm{sec}$ and $65^{\circ} \mathrm{C}$ for $45 \mathrm{sec}$. U6 small nuclear RNA and GAPDH served as the endogenous controls for miR-584 and CCND1 mRNA, respectively. Data were analysed using the $2^{-\Delta \Delta C q}$ method (19). The primers were designed as follows: miR-584, forward 5'-TGCAATGTGTGT GTTAGCCA-3', reverse 5'-ATCATTGCTCCTTGGATG GT-3'; U6, forward 5'-CTCGCTTCGGCAGCACA-3', reverse 5'-AACGCTTCACGAATTTGCGT-3'; CCND1, forward 5'-GACGGCCGAGAAGCTGTGCA-3', reverse 5'- GCCACC ATGGAGGGCGGATT-3'; and GAPDH, forward 5'-CGG AGTCAACGGATTTGGTCGTAT-3', reverse 5'-AGCCTT CTCCATGGTGGTGAAGAC-3'.

Cell Counting Kit-8 (CCK-8) assay. Transfected cells were collected at $24 \mathrm{~h}$ post-transfection and were inoculated into 96-well plates at a density of 3,000 cells/well. Following culturing for $0,24,48$ and $72 \mathrm{~h}, \mathrm{CCK}-8$ assays were performed to determine cell proliferation. In total, $10 \mu 1$ CCK-8 reagent (Sigma-Aldrich; Merck KGaA, Darmstadt, Germany) was added into each well at each time point. The cells were further incubated at $37^{\circ} \mathrm{C}$ under $5 \% \mathrm{CO}_{2}$ for $2 \mathrm{~h}$, and the absorbance of each well was detected at $450 \mathrm{~nm}$ wavelength using a microplate reader (Bio-Rad Laboratories, Inc., Hercules, CA, USA).

Matrigel invasion assay. The invasive ability of transfected cells was evaluated using 24-well Transwell chambers that were precoated with Matrigel (Becton, Dickinson and Company, Franklin Lakes, NJ, USA). Transfected cells were incubated for $48 \mathrm{~h}$ and subsequently harvested and washed twice with FBS-free DMEM. A total of $1 \times 10^{5}$ transfected cells, suspended in FBS-free DMEM, were added to the upper Transwell chambers. In total, $500 \mu$ l DMEM containing 20\% FBS was added to the lower chambers as a chemoattractant. Following $24 \mathrm{~h}$ of incubation at $37^{\circ} \mathrm{C}$ with $5 \% \mathrm{CO}_{2}$, cotton swabs were used to remove the non-invasive cells remaining on the upper surface of the membranes. The invasive cells were fixed with $100 \%$ methanol at room temperature for $20 \mathrm{~min}$ and stained with $0.1 \%$ crystal violet at room temperature for $20 \mathrm{~min}$. The number of invasive cells was counted in five randomly chosen fields under an inverted light (Olympus Corporation, Tokyo, Japan; magnification, x200). 
Bioinformatics prediction. TargetScan (http://www.targetscan. org) and Pictar (http://www.pictar.org/) were used to predict the potential targets of miR-584.

Luciferase reporter gene assay. The 3'-UTRs of CCND1 containing the wild-type (WT) or mutant (Mut) target sequences of miR-584 were amplified by Shanghai GenePharma Co., Ltd. (Shanghai, China), inserted downstream of the pMIR-REPORT ${ }^{\mathrm{TM}}$ Luciferase Plasmid (Thermo Fisher Scientific, Inc.) and defined as WT-CCND1-3'-UTR and Mut-CCND1-3'-UTR, respectively. For reporter assays, WT-CCND1-3'-UTR or Mut-CCND1-3'-UTR, together with miR-584 mimics or miR-NC were transfected into Panc-1 and Sw1990 cells using Lipofectamine ${ }^{\circledR} 2000$ reagent. Luciferase activity was determined $48 \mathrm{~h}$ following transfection using the Dual-Luciferase ${ }^{\circledR}$ Reporter Assay System (Promega Corporation, Madison, WI, USA) according to the manufacturer's protocol. Firefly luciferase activity was normalised to Renilla luciferase activity.

Western blot analysis. Tissue specimens or cells were lysed in cold radioimmunoprecipitation assay buffer (Thermo Fisher Scientific, Inc.) to isolate the total protein, which was subsequently quantified using the Bicinchoninic Acid Protein Assay kit (Pierce; Thermo Fisher Scientific, Inc.) according to the manufacturer's protocol. Subsequently, equal amounts of protein $(30 \mu \mathrm{g})$ were separated by $10 \%$ SDS-PAGE and transferred onto polyvinylidene difluoride membranes, followed by blocking with $5 \%$ skimmed milk that was dissolved in Tris-buffered saline containing $0.1 \%$ Tween-20 (TBST) for $2 \mathrm{~h}$ at room temperature. The membranes were incubated overnight at $4^{\circ} \mathrm{C}$ with rabbit anti-human CCND1 monoclonal antibody (1:1,000; cat. no. ab16663; Abcam, Cambridge, UK) or rabbit anti-human GAPDH monoclonal antibody $(1: 1,000$; cat. no. ab128915; Abcam). The membranes were washed three times with TBST and subsequently probed with goat anti-rabbit horseradish peroxidase-conjugated immunoglobulin G secondary antibody (1:5,000; cat. no. ab205718; Abcam) at room temperature for $2 \mathrm{~h}$. Chemiluminescence detection was performed using an enhanced chemiluminescence kit (Thermo Fisher Scientific, Inc.). GAPDH served as the loading control. Quantity One software version 4.62 (Bio-Rad Laboratories, Inc., Hercules, CA, USA) was used to analyse the densitometry.

Statistical analysis. Data are presented as the mean \pm standard deviation from at least three independent experiments and analysed using SPSS software (version 19.0; IBM Corp., Armonk, NY, USA). Student's t-test and one-way analysis of variance (ANOVA) were used to compare the differences between groups. Student-Newman-Keuls test was used as the post hoc test following ANOVA. $\mathrm{P}<0.05$ was considered to indicate a statistically significant difference.

\section{Results}

miR-584 expression is decreased in pancreatic cancer tissues and cell lines. To measure the miR-584 expression level in pancreatic cancer, RT-qPCR was performed in 26 primary pancreatic cancer tissues and their matched normal adjacent specimens. miR-584 expression was significantly downregulated in pancreatic cancer tissues compared with the normal adjacent specimens (Fig. 1A). The expression level of miR-584 was additionally determined in three human pancreatic cancer cell lines (Panc-1, Sw1990 and Bxpc-3). As depicted in Fig. 1B, the miR-584 expression level was significantly decreased in all three pancreatic cancer cell lines compared with the normal human pancreatic cell line HPDE6c7. Therefore, these findings suggested that miR-584 may be associated with the development of pancreatic cancer.

miR-584 overexpression suppresses the proliferation and invasion of pancreatic cancer cells. The significant decrease in miR-584 expression level in pancreatic cancer suggested that miR-584 may serve tumour suppressive roles in the progression of pancreatic cancer. To test this hypothesis, Panc-1 and Sw1990 cell lines that expressed relatively lower miR-584 expression were selected for the following functional experiments. miR-584 mimics or miR-NC was transfected into Panc-1 and Sw1990 cells, and RT-qPCR analysis was used to evaluate the transfection efficiency. miR-584 expression was significantly upregulated in Panc-1 and Sw1990 cells transfected with miR-584 mimics compared with cells transfected with miR-NC (Fig. 2A). CCK-8 and Matrigel invasion assays were performed to examine the effects of miR-584 overexpression on cell proliferation and invasion in pancreatic cancer, respectively. miR-584 overexpression resulted in a significant decrease in the proliferation at 48 and $72 \mathrm{~h}$ (Fig. 2B) and invasion (Fig. 2C) of Panc-1 and Sw1990 cells. The present results suggested that miR-584 may serve as a tumour suppressor in pancreatic cancer.

CCND1 is a direct target gene of miR-584 in pancreatic cancer cells. The mechanism underlying the action of miR-584 in pancreatic cancer was further examined. Bioinformatics analysis was conducted to predict the potential targets of miR-584. CCND1, a predicted target of miR-584 (Fig. 3A), serves crucial roles in pancreatic cancer progression (20-25) and was thus selected for further investigation. To test whether the 3'-UTR of CCND1 is directly targeted by miR-584, luciferase reporter plasmids were constructed and co-transfected into Panc-1 and Sw1990 cells together with miR-584 mimics or miR-NC. The overexpression of miR-584 significantly decreased the luciferase activity of WT-CCND1-3'-UTR; however, had no effect on Mut-CCND1-3'-UTR in Panc-1 and Sw1990 cells (Fig. 3B). To test whether miR-584 may affect endogenous CCND1 expression, RT-qPCR and western blot analysis were performed to measure CCND1 mRNA and protein expression levels, respectively, in Panc-1 and Sw1990 cells that were transfected with miR-584 mimics or miR-NC. The overexpression of miR-584 significantly decreased CCND1 mRNA (Fig. 3C) and protein (Fig. 3D) expression levels in Panc-1 and Sw1990 cells. The present results demonstrated that CCND1 was directly targeted by miR-584 in pancreatic cancer cells.

CCND1 knockdown mimics the suppressive effect of miR-584 in pancreatic cancer cells. To elucidate the roles of CCND1 in pancreatic cancer, a knockdown of endogenous CCND1 expression was performed in Panc-1 and Sw1990 cells via transfection with si-CCND1. Western blot analysis demonstrated that the 

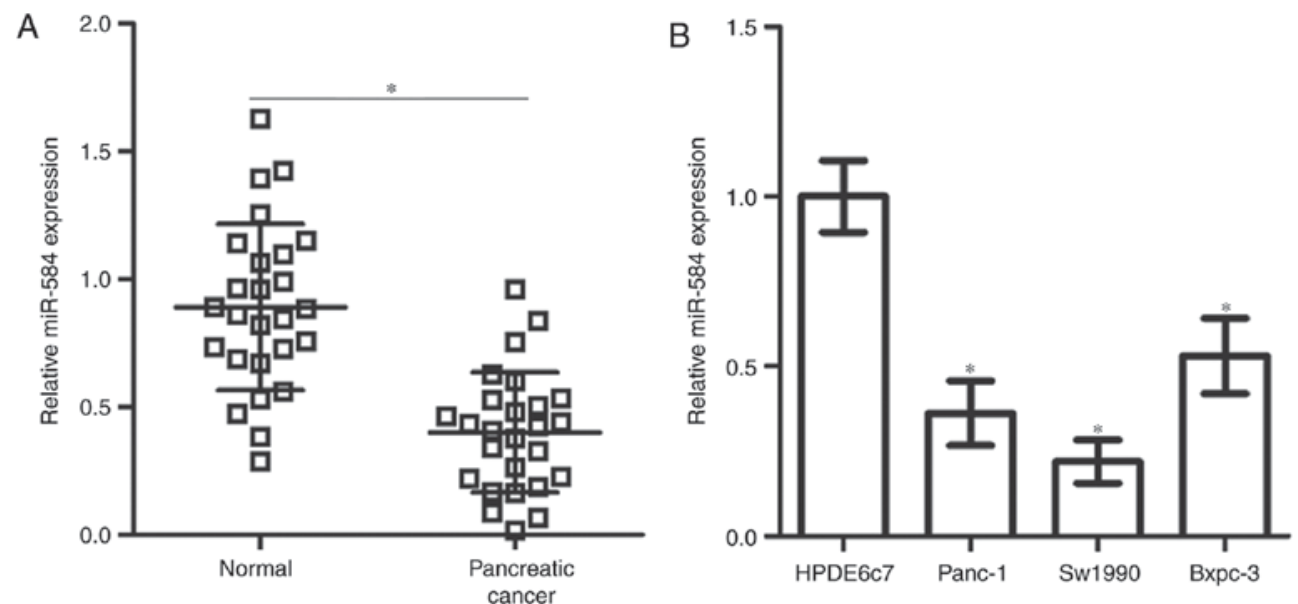

Figure 1. Expression levels of miR-584 in pancreatic cancer tissues and cell lines. (A) Total RNA was isolated from 26 primary pancreatic cancer tissues and their matched normal adjacent specimens. RT-qPCR was performed to detect miR-584 expression. ${ }^{*} \mathrm{P}<0.05$. (B) Expression levels of miR-584 in three human pancreatic cancer cell lines (Panc-1, Sw1990 and Bxpc-3) and a normal human pancreatic cell line HPDE6c7 were determined by RT-qPCR. "P<0.05 vs. HPDE6c7. miR-584, microRNA-584-5p; RT-qPCR, reverse transcription-quantitative polymerase chain reaction.
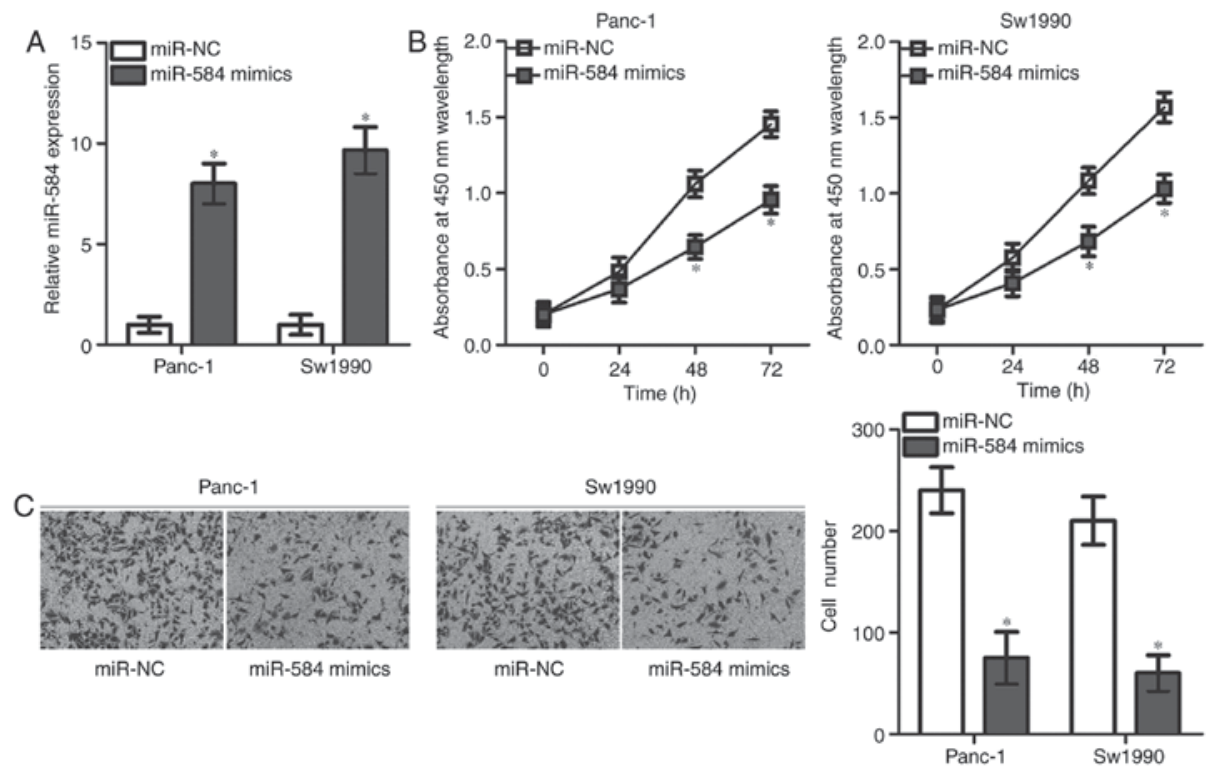

Figure 2. Effects of miR-584 overexpression on the proliferation and invasion of Panc-1 and Sw1990 cells. (A) miR-584 mimics or miR-NC was transfected into Panc-1 and Sw1990 cells, and the transfection efficiency was evaluated by reverse transcription-quantitative polymerase chain reaction. (B) Proliferation of Panc-1 and Sw1990 cells that were transfected with miR-584 mimics or miR-NC was determined by a Cell Counting Kit-8 assay. (C) Transwell invasion assay was performed to detect the invasive ability of Panc-1 and Sw1990 cells following transfection with miR-584 mimics or miR-NC (magnification, x200). "P<0.05 vs. respective miR-NC. miR, microRNA; NC, negative control.

CCND1 protein was significantly downregulated in Panc-1 and Sw1990 cells following transfection with si-CCND1 (Fig. 4A). Functional experiments indicated that the inhibition of CCND1 decreased the proliferative (Fig. 4B and C) and invasive ability (Fig. 4D) of Panc-1 and Sw1990 cells, mimicking miR-584 overexpression. The present results suggested that miR-584 directly targeted CCND1 in pancreatic cancer cells.

CCND1 overexpression attenuates the suppressive roles of miR-584 in pancreatic cancer cells. Rescue experiments were performed to test the role of CCND1 in mediating the action of miR-584 in pancreatic cancer cells. Panc-1 and Sw1990 cells were transfected with miR-584 mimics, in combination with pCMV-CCND1 or empty pCMV plasmids. Western blot analysis demonstrated that the CCND1 overexpressing plasmid pCMV-CCND1 was able to effectively restore the CCND1 protein expression level in Panc-1 and Sw1990 cells (Fig. 5A). In addition, CCK-8 and Matrigel invasion assays demonstrated that restoring CCND1 expression inhibited the suppressive effects of miR-584 overexpression on the proliferation (Fig. 5B and C) and invasion (Fig. 5D) of Panc-1 and Sw1990 cells. The present results suggested that miR-584 served tumour suppressing roles in pancreatic cancer cells by repressing CCND1 expression.

\section{Discussion}

Multiple previous studies have demonstrated that the dysregulation of miRNAs is implicated in the occurrence 


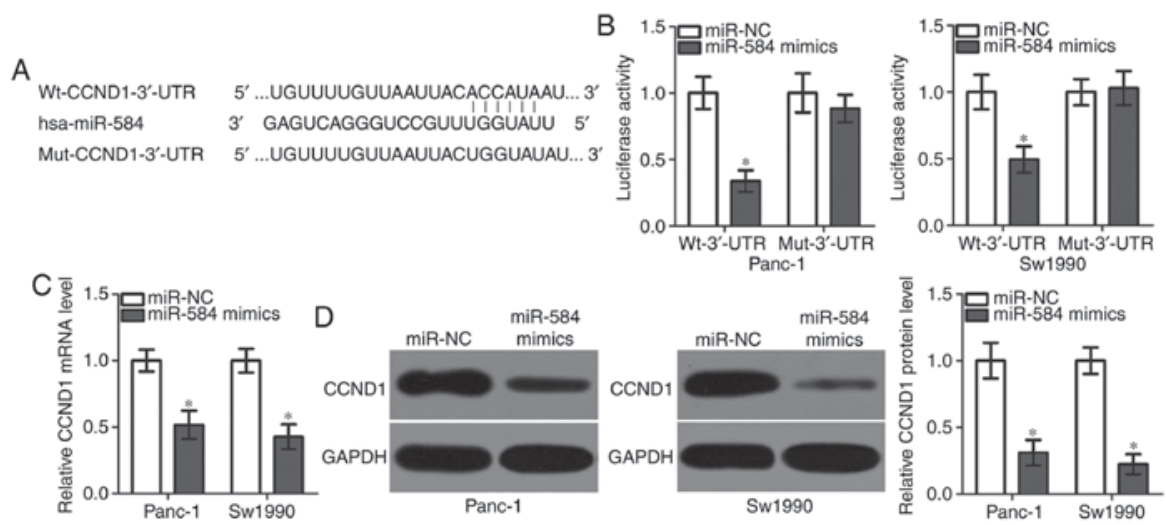

Figure 3. miR-584 inhibits the mRNA and protein expression levels of CCND1 by directly binding to its 3'-UTR in Panc-1 and Sw1990 cells. (A) Predicted WT binding sequences for miR-584 in the 3'-UTR of CCND1 and the mutations in the binding sequences. (B) Luciferase activity in Panc-1 and Sw1990 cells co-transfected with WT-CCND1-3'-UTR or Mut-CCND1-3'-UTR luciferase reporter plasmid and miR-584 mimics or miR-NC was detected using the Dual-Luciferase ${ }^{\circledast}$ Reporter Assay System. (C) mRNA and (D) protein expression levels of CCND1 in Panc-1 and Sw1990 cells with miR-584 mimics or miR-NC transfection were measured by reverse transcription-quantitative polymerase chain reaction and western blot analysis, respectively. $\mathrm{P}<0.05$ vs. respective miR-NC. 3'-UTR, 3'-untranslated region; CCND1, cyclin D1; miR, microRNA; Mut, mutant; NC, negative control; WT, wild-type.
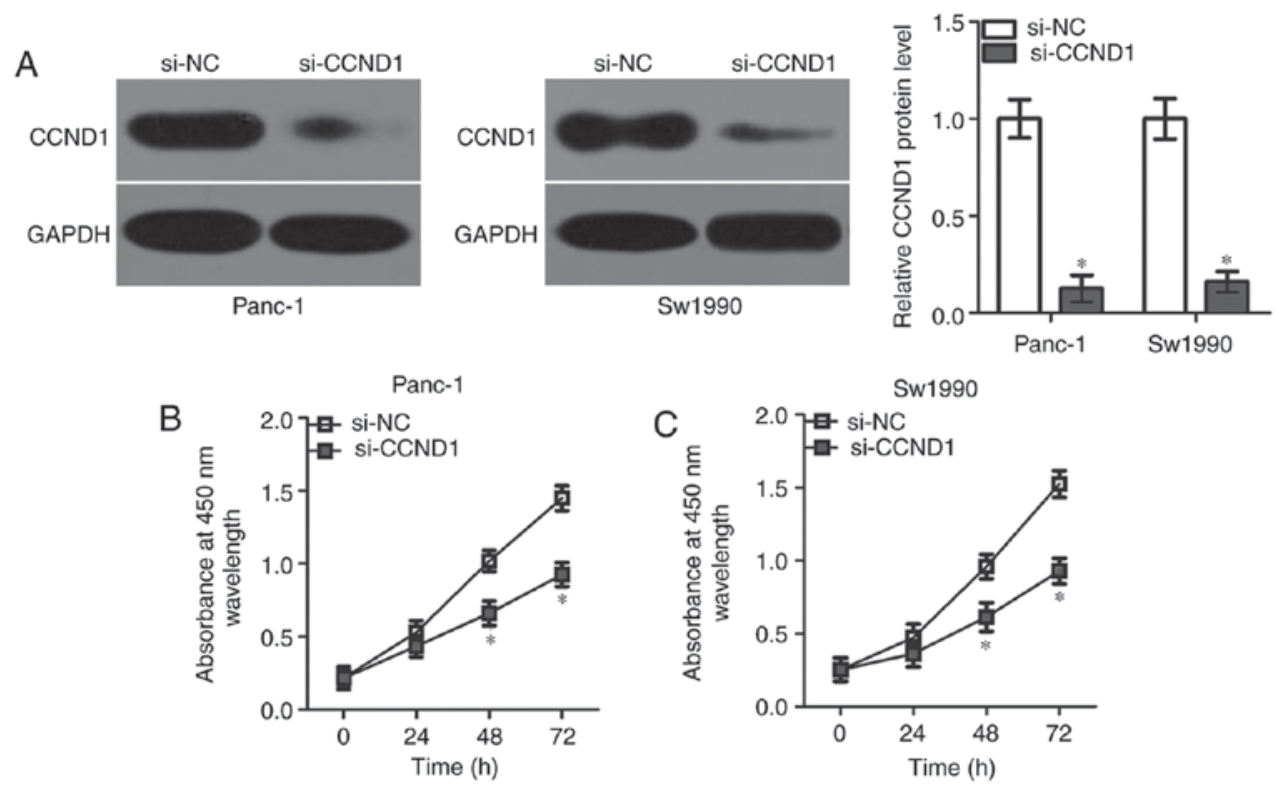

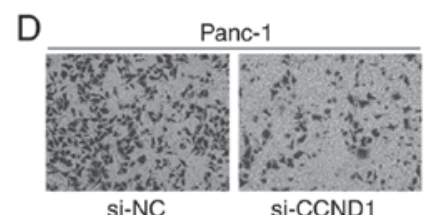

si-NC

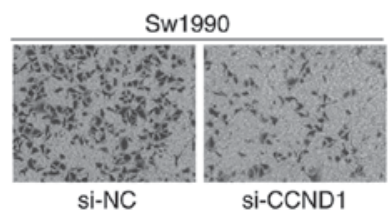

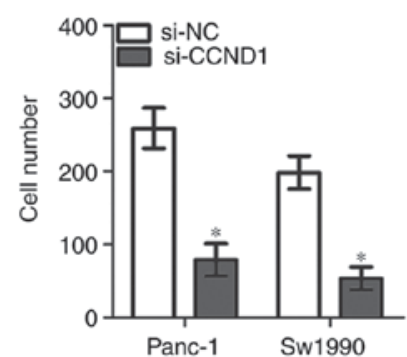

Figure 4. CCND1 knockdown attenuates the proliferation rate and invasive ability of Panc-1 and Sw1990 cells. (A) CCND1 protein expression in Panc-1 and Sw1990 cells transfected with si-CCND1 or si-NC was assayed by western blot analysis. Proliferation of (B) Panc-1 and (C) Sw1990 cells transfected with si-CCND1 or si-NC was assessed by Cell Counting Kit-8 assays, and (D) invasion of Panc-1 and Sw1990 cells transfected with si-CCND1 or si-NC was assessed by Matrigel invasion assays. Magnification, x200. ${ }^{*} \mathrm{P}<0.05$ vs. si-NC. CCND1, cyclin D1; NC, si-negative control; si, small interfering RNA.

and development of pancreatic cancer (26-28). Nevertheless, the detailed roles of miRNAs in pancreatic cancer and their underlying mechanisms of action remain largely unknown. Hence, the further characterization of deregulated miRNAs in pancreatic cancer may provide insight into the oncogenesis and progression of pancreatic cancer, and guide the identification of novel therapeutic targets for treating patients with this disease. In the present study, the expression, biological roles and underlying mechanisms of miR-584 in pancreatic cancer were examined. The expression of miR-584 in pancreatic 

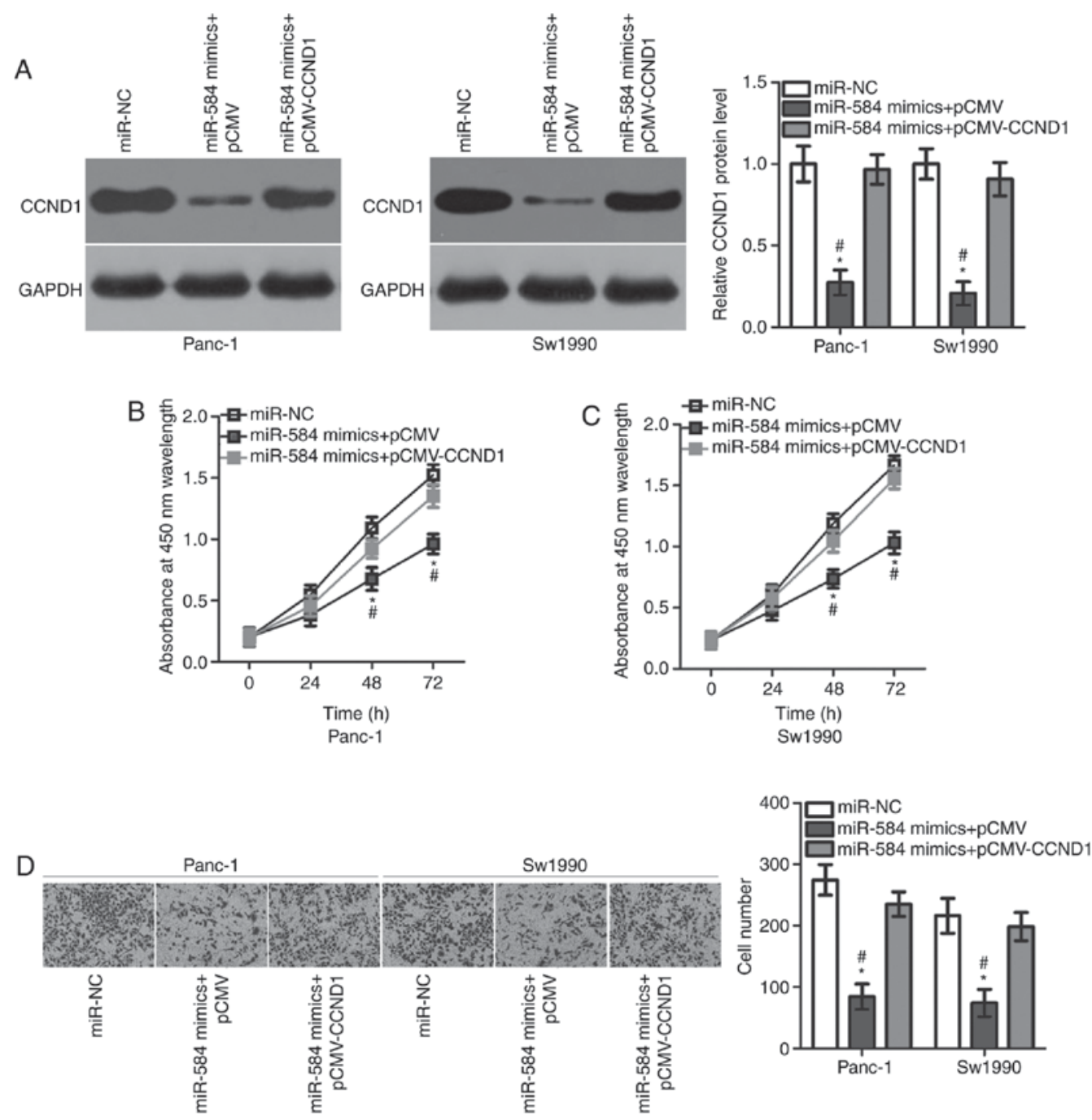

Figure 5. Restoration of CCND1 abolishes the suppressive roles of miR-584 overexpression on the proliferation and invasion of Panc-1 and Sw1990 cells. (A) CCND1 protein expression level of indicated cells was measured by western blot analysis. Proliferation rate of the (B) Panc-1 and (C) Sw1990 cells was measured by Cell Counting Kit-8 assays, and (D) invasive ability of the Panc-1 and Sw1990 cells was assessed by Matrigel invasion assays. Magnification, x200. "P<0.05 vs. miR-NC; "P<0.05 vs. miR-584 mimics+pCMV-CCND1. CCND1, cyclin D1; miR, microRNA; NC, negative control; pCMV, cytomegalovirus promoter.

cancer was investigated and it was identified that the miR-584 expression level was significantly decreased in pancreatic cancer tissues and cell lines. Notably, ectopic miR-584 expression decreased the proliferation and invasion of pancreatic cancer cells and CCND1 was identified as a direct target gene of miR-584 in pancreatic cancer cells. It was observed that inhibition of CCND1 and miR-584 overexpression led to similar effects on pancreatic cancer cells. Importantly, restoring CCND1 expression inhibited the suppressive roles of miR-584 overexpression in pancreatic cancer cells. The present results demonstrated that miR-584 inhibited pancreatic cancer progression by directly targeting CCND1, suggesting that this miRNA may represent a novel potential therapeutic target for the treatment of patients with pancreatic cancer.

miR-584 has been identified to be differently expressed in numerous types of human malignancy. miR-584 expression was downregulated in non-small cell lung cancer, and its downregulation was strongly associated with tumour size, tumour node, metastasis stage and distant metastasis (16). miR-584 expression was decreased in neuroblastoma tissues and cell lines, and was an independent prognostic biomarker for predicting the prognosis of patients with neuroblastoma (17). In gastric cancer, low expression of miR-584 was detected and its expression was negatively correlated with tumour size (18). Aberrant downregulation of miR-584 was additionally reported in glioma (29) and clear cell renal cell carcinoma (30). Collectively, numerous findings suggested that miR-584 may be a valuable biomarker for the diagnosis and prediction of the therapeutic outcomes of patients with these specific cancer types.

miR-584 serves tumour suppressive roles in multiple types of human cancer. Restoration of miR-584 expression suppressed the proliferative and invasive capabilities of non-small cell lung cancer cells (16). Xiang et al (17) observed that miR-584 overexpression prohibited the proliferation, metastasis and angiogenesis of neuroblastoma cells in vitro and in vivo. Li et al (18) demonstrated that the upregulation of miR-584 expression inhibited gastric cancer cell proliferation and induced apoptosis in vitro. Wang et al (29) demonstrated that miR-584 restoration supressed cell growth and motility, and induced apoptosis of glioma. Ueno et al (30) demonstrated 
that miR-584 overexpression attenuated cell migration and invasion in clear cell renal cell carcinoma. These findings suggested that miR-584 may serve crucial roles in the tumourigenesis and tumour development of these human cancer types. Therefore, miR-584 restoration may be an attractive therapeutic strategy for treating patients with these specific human cancer types.

Identifying the direct targets of miR-584 may elucidate the mechanisms underlying its biological roles in human cancer. Numerous target genes of miR-584 have been previously identified, including metadherin (16) in non-small cell lung cancer, matrix metalloproteinase-14 (17) in neuroblastoma, WW domain containing E3 ubiquitin protein ligase 1 (18) in gastric cancer, pituitary tumour-transforming gene 1 (29) in glioma and Rho-associated protein kinase 1 (30) in clear cell renal cell carcinoma. The CCND1 gene, located on chromosome 11q13, was demonstrated to be a direct target gene of miR-584 in pancreatic cancer. It is highly expressed in numerous types of human cancer, including colorectal cancer (31), breast cancer (32), lung cancer (33), hepatocellular carcinoma (34) and glioma (35). CCND1 expression was upregulated in pancreatic cancer tissues and cell lines and the high expression of CCND1 was correlated with differentiation and prognosis of patients with pancreatic cancer $(20,21)$. Additionally, the dysregulation of CCND1 is implicated in the development of pancreatic cancer by affecting cell growth and metastasis in vitro and in vivo (22-25). The present results suggested that the miR-584/CCND1 pathway has potential therapeutic applications in treating patients with this malignancy.

In conclusion, miR-584 was downregulated in pancreatic cancer tissues and cell lines. miR-584 may have tumour suppressive roles in pancreatic cancer partly by directly targeting CCND1, suggesting that this miRNA may represent a promising therapeutic target for treating patients with this aggressive disease. However, the association between miR-584 and the prognosis of patients with pancreatic cancer was not analysed, representing a limitation of the present study, that requires investigation in future studies.

\section{Acknowledgements}

Not applicable.

\section{Funding}

The present study was supported by the Heilongjiang Natural Science Foundation (China; grant no. H2017072) and Key Topics of Jiamusi University (Jiamusi, China; grant no. Sz2013-006).

\section{Availability of data and materials}

The datasets used and/or analysed during the present study are available from the corresponding author on reasonable request.

\section{Authors' contributions}

YQ, GC and MH designed the study. GC, MH, XQ and KW performed the functional assays. KW analysed the data. All authors read and approved the final manuscript.

\section{Ethics approval and consent to participate}

The present study was approved by the Research Ethics Committee of The First Affiliated Hospital of Jiamusi University (Jiamusi, China) and was performed in accordance with the Declaration of Helsinki and the guidelines of the Ethics Committee of The First Affiliated Hospital of Jiamusi University. Written informed consent was obtained from all patients for the use of their clinical tissues.

\section{Patient consent for publication}

Not applicable.

\section{Competing interests}

The authors declare that they have no competing interests.

\section{References}

1. Chen W, Zheng R, Zhang S, Zhao P, Li G, Wu L and He J: Report of incidence and mortality in China cancer registries, 2009. Chin J Cancer Res 25: 10-21, 2013

2. Kamisawa T, Wood LD, Itoi T and Takaori K: Pancreatic cancer. Lancet 388: 73-85, 2016.

3. Cameron JL, Riall TS, Coleman J and Belcher KA: One thousand consecutive pancreaticoduodenectomies. Ann Surg 244: 10-15, 2006.

4. Niedergethmann M, Alves F, Neff JK, Heidrich B, Aramin N, Li L, Pilarsky C, Grützmann R, Allgayer H, Post S and Gretz N: Gene expression profiling of liver metastases and tumour invasion in pancreatic cancer using an orthotopic SCID mouse model. Br J Cancer 97: 1432-1440, 2007.

5. Feng H, Wang Y, Su J, Liang H, Zhang CY, Chen X and Yao W: MicroRNA-148a suppresses the proliferation and migration of pancreatic cancer cells by down-regulating ErbB3. Pancreas 45: 1263-1271, 2016.

6. Lewis BP, Burge CB and Bartel DP: Conserved seed pairing, often flanked by adenosines, indicates that thousands of human genes are microRNA targets. Cell 120: 15-20, 2005.

7. Galasso M, Sandhu SK and Volinia S: MicroRNA expression signatures in solid malignancies. Cancer J 18: 238-243, 2012.

8. Wald P, Liu XS, Pettit C, Dillhoff M, Manilchuk A, Schmidt C, Wuthrick E, Chen W and Williams TM: Prognostic value of microRNA expression levels in pancreatic adenocarcinoma: A review of the literature. Oncotarget 8: 73345-73361, 2017.

9. Sekar D, Krishnan R, Thirugnanasambantham K, Rajasekaran B, Islam VI and Sekar P: Significance of microRNA 21 in gastric cancer. Clin Res Hepatol Gastroenterol 40: 538-545, 2016.

10. Celano M, Rosignolo F, Maggisano V, Pecce V, Iannone M, Russo D and Bulotta S: MicroRNAs as biomarkers in thyroid carcinoma. Int J Genomics 2017: 6496570, 2017.

11. Fadejeva I, Olschewski H and Hrzenjak A: MicroRNAs as regulators of cisplatin-resistance in non-small cell lung carcinomas. Oncotarget 8: 115754-115773, 2017.

12. Ebert MS and Sharp PA: Roles for microRNAs in conferring robustness to biological processes. Cell 149: 515-524, 2012.

13. Wang Z, Tan Y, Yu W, Zheng S, Zhang S, Sun L and Ding K: Small role with big impact: MiRNAs as communicators in the cross-talk between cancer-associated fibroblasts and cancer cells. Int J Biol Sci 13: 339-348, 2017.

14. Kim YK, Yu J, Han TS, Park SY, Namkoong B, Kim DH, Hur K, Yoo MW, Lee HJ, Yang HK and Kim VN: Functional links between clustered microRNAs: Suppression of cell-cycle inhibitors by microRNA clusters in gastric cancer. Nucleic Acids Res 37: 1672-1681, 2009.

15. Fu F, Wan X, Wang D, Kong Z, Zhang Y, Huang W, Wang C, Wu H and Li Y: MicroRNA-19a acts as a prognostic marker and promotes prostate cancer progression via inhibiting VPS37A expression. Oncotarget 9: 1931-1943, 2017.

16. Zhang Y, Wang Y and Wang J: MicroRNA-584 inhibits cell proliferation and invasion in non-small cell lung cancer by directly targeting MTDH. Exp Ther Med 15: 2203-2211, 2018. 
17. Xiang X, Mei H, Qu H, Zhao X, Li D, Song H, Jiao W, Pu J, Huang K, Zheng L and Tong Q: miRNA-584-5p exerts tumor suppressive functions in human neuroblastoma through repressing transcription of matrix metalloproteinase 14 . Biochim Biophys Acta 1852: 1743-1754, 2015.

18. Li Q, Li Z, Wei S, Wang W, Chen Z, Zhang L, Chen L, Li B, Sun G, $\mathrm{Xu} \mathrm{J}$, et al: Overexpression of miR-584-5p inhibits proliferation and induces apoptosis by targeting WW domain-containing E3 ubiquitin protein ligase 1 in gastric cancer. J Exp Clin Cancer Res 36: 59, 2017.

19. Livak KJ and Schmittgen TD: Analysis of relative gene expression data using real-time quantitative PCR and the 2(-Delta Delta C (T)) method. Methods 25: 402-408, 2001.

20. Gansauge S, Gansauge F, Ramadani M, Stobbe H, Rau B, Harada N and Beger HG: Overexpression of cyclin D1 in human pancreatic carcinoma is associated with poor prognosis. Cancer Res 57: 1634-1637, 1997.

21. Li YJ and Ji XR: Relationship between the expression of beta-cat, cyclin D1 and c-myc and the occurance and biological behavior of pancreatic cancer. Zhonghua Bing Li Xue Za Zhi 32: 238-241, 2003 (In Chinese).

22. Xu Y, Liu T and Gao J: Effects of antisense cyclin D1 expressing vector on the cell growth and apoptosis of pancreatic carcinoma. Zhonghua Bing Li Xue Za Zhi 27: 348-351, 1998 (In Chinese).

23. Yan L, Wang Y, Wang ZZ, Rong YT, Chen LL, Li Q, Liu T Chen YH, Li YD, Huang ZH and Peng J: Cell motility and spreading promoted by CEACAM6 through cyclin D1/CDK4 in human pancreatic carcinoma. Oncol Rep 35: 418-426, 2016.

24. Lee Y, Ko D, Min HJ, Kim SB, Ahn HM, Lee Y and Kim S: TMPRSS4 induces invasion and proliferation of prostate cancer cells through induction of Slug and cyclin D1. Oncotarget 7: 50315-50332, 2016.

25. Zhang Y, Su Y, Zhao Y, Lv G and Luo Y: MicroRNA720 inhibits pancreatic cancer cell proliferation and invasion by directly targeting cyclin D1. Mol Med Rep 16: 9256-9262, 2017.

26. Cao W, Jin H, Zhang L, Chen X and Qian H: Identification of miR-601 as a novel regulator in the development of pancreatic cancer. Biochem Biophys Res Commun 483: 638-644, 2017.
27. Le Large TY, Meijer LL, Prado MM, Kazemier G, Frampton AE and Giovannetti E: Circulating microRNAs as diagnostic biomarkers for pancreatic cancer. Expert Rev Mol Diagn 15: $1525-1529,2015$

28. Takikawa T, Masamune A, Yoshida N, Hamada S, Kogure T and Shimosegawa T: Exosomes derived from pancreatic stellate cells: MicroRNA signature and effects on pancreatic cancer cells. Pancreas 46: 19-27, 2017

29. Wang XP, Deng XL and Li LY: MicroRNA-584 functions as a tumor suppressor and targets PTTG1IP in glioma. Int J Clin Exp Pathol 7: 8573-8582, 2014.

30. Ueno K, Hirata H, Shahryari V, Chen Y, Zaman MS, Singh K, Tabatabai ZL, Hinoda Y and Dahiya R: Tumour suppressor microRNA-584 directly targets oncogene Rock-1 and decreases invasion ability in human clear cell renal cell carcinoma. Br J Cancer 104: 308-315, 2011.

31. Li Y, Wei J, Xu C, Zhao Z and You T: Prognostic significance of cyclin D1 expression in colorectal cancer: A meta-analysis of observational studies. PLoS One 9: e94508, 2014.

32. Li X, Huo X, Li W, Yang Q, Wang Y and Kang X: Genetic association between cyclin D1 polymorphism and breast cancer susceptibility. Tumour Biol 35: 11959-11965, 2014.

33. Betticher DC, Heighway J, Hasleton PS, Altermatt HJ, Ryder WD, Cerny $\mathrm{T}$ and Thatcher N: Prognostic significance of CCND1 (cyclin D1) overexpression in primary resected non-small-cell lung cancer. Br J Cancer 73: 294-300, 1996.

34. Lu JW, Lin YM, Chang JG, Yeh KT, Chen RM, Tsai JJ, Su WW and $\mathrm{Hu}$ RM: Clinical implications of deregulated CDK4 and Cyclin D1 expression in patients with human hepatocellular carcinoma. Med Oncol 30: 379, 2013.

35. Xu Z, Zeng X, Tian D, Xu H, Cai Q, Wang J and Chen Q: MicroRNA-383 inhibits anchorage-independent growth and induces cell cycle arrest of glioma cells by targeting CCND1. Biochem Biophys Res Commun 453: 833-838, 2014. 